\title{
Adiabatic compression of terahertz waves using metal flares
}

\author{
Michael Theuer, ${ }^{1,2}$ René Beigang, ${ }^{2,3}$ and Daniel R. Grischkowsky ${ }^{1, a)}$ \\ ${ }^{1}$ School of Electrical and Computer Engineering, Oklahoma State University, Stillwater, \\ Oklahoma 74078, USA \\ ${ }^{2}$ Department of Terahertz, Measurement and Systems, Fraunhofer Institute for Physical Measurement \\ Techniques, 67663 Kaiserslautern, Rhineland-Palatinate, Germany \\ ${ }^{3}$ Department of Physics, University of Kaiserslautern, 67663 Kaiserslautern, Rhineland-Palatinate, \\ Germany
}

(Received 19 February 2010; accepted 27 April 2010; published online 14 May 2010)

\begin{abstract}
We report on the adiabatic free-space coupling of broadband terahertz $(\mathrm{THz})$ radiation through a subwavelength gap using metal flares. Amplitude coupling ratios better than $30 \%$ are obtained by optimizing the curvature. This scheme shows a large potential for increasing the sensitivity in $\mathrm{THz}$ spectroscopy using high spatial confinement without the need of transmission optics or high precision components. The intrinsic curvature of metal sheets is already sufficient to carry out experiments downsizing the microwave approach. (C) 2010 American Institute of Physics.
\end{abstract}

[doi:10.1063/1.3430740]

Terahertz $(\mathrm{THz})$ radiation has proven to offer a large potential in the field of sensing and identification of chemical substances. ${ }^{1-3}$ Almost all molecules show spectral characteristics in the $\mathrm{THz}$ frequency range, depending on the size of the molecule and the particular crystal structure. Terahertz time-domain spectroscopy (THz-TDS) based on femtosecond laser pulses is a very powerful tool, if a large spectral bandwidth is needed. Typical THz-TDS systems address a spectral bandwidth extending from $100 \mathrm{GHz}$ to $4 \mathrm{THz}$.

Parallel-plate waveguides can be used to increase the sensitivity of the THz-TDS to thin layers of material. Their big advantage is the high spatial compression of the $\mathrm{THz}$ wave with respect to the ratio of the waveguide plate separation to the wavelength. ${ }^{4}$ This, together with the high filling factor even for a tiny amount of sample, leads to higher sensitivity with a factor given by $\Gamma=\mathrm{L} / \mathrm{n}^{3} \mathrm{~b}$, with $\mathrm{L}$ the length of the waveguide, $n$ the refractive index of the sample, and $\mathrm{b}$ the plate separation. ${ }^{5}$ For a typical parallel-plate waveguide, shown in Fig. 1, cylindrical silicon lenses are used to focus the THz spot size from $1 \mathrm{~cm}$ down to a line focus of $150 \mu \mathrm{m}$ at the entrance face. ${ }^{6}$ As the plate separation is typically smaller (100 $\mu \mathrm{m}$ or less), coupling losses occur at the input and output of the waveguide.

Consequently, increasing the sensitivity factor $\Gamma$ by reducing the plate separation $b$, also reduces the coupling efficiency of the incoming $\mathrm{THz}$ pulse to the waveguide. Various approaches have been introduced to overcome this problem. Previous results have shown that the adiabatic compression of the TEM mode within a waveguide is highly efficient, by reducing the input plate separation $\mathrm{b}$ from $100 \mu \mathrm{m}$ at the entrance face to $10 \mu \mathrm{m}$ at midwaveguide, and then increasing to $100 \mu \mathrm{m}$ at the output face. ${ }^{7}$ As recently shown by Kim et $a l .{ }^{8}$ high precision milled flares can be used to couple and guide free-space $\mathrm{THz}$ radiation through a parallel-plate waveguide, thereby eliminating the coupling lenses. Our conceptually similar approach in this paper is based on using standard $100 \mu \mathrm{m}$ thick copper shim (cut from a commercial $9 \mathrm{~cm}$ diameter shim stock roll using a paper cutter) to make the coupling flares. This is advantageous in terms of simplic-

${ }^{a)}$ Electronic mail: daniel.grischkowsky@okstate.edu. ity and availability, especially for real-world applications.

The experimental setup (Fig. 1) consists of a standard THz-TDS system. ${ }^{9}$ Two off-axis parabolic mirrors collimate the $\mathrm{THz}$ beam forming a beamwaist with diameter proportional to wavelength $(9 \mathrm{~mm}$ at $1 \mathrm{THz})$ in a confocal geometry. In Fig. 1(a) the setup of a parallel-plate waveguide system for high-resolution spectroscopy of thin layers of material is shown. ${ }^{4}$ The standard way to couple the $\mathrm{THz}$ radiation into the subwavelength gap of the waveguide uses cylindrical Si lenses. Let the coupling ratio be defined as the ratio of amplitudes of the $\mathrm{THz}$ electric field measured with the waveguide assembly in the beam normalized to the amplitude without it (free-space reference). However, in this quasioptical approach, the transmission suffers from Fresnel reflection losses for the inserted two $\mathrm{Si}$ lenses $\left(\mathrm{n}_{\mathrm{Si}}=3.42\right)$. This accounts for a coupling ratio reduction of 0.5 corre-

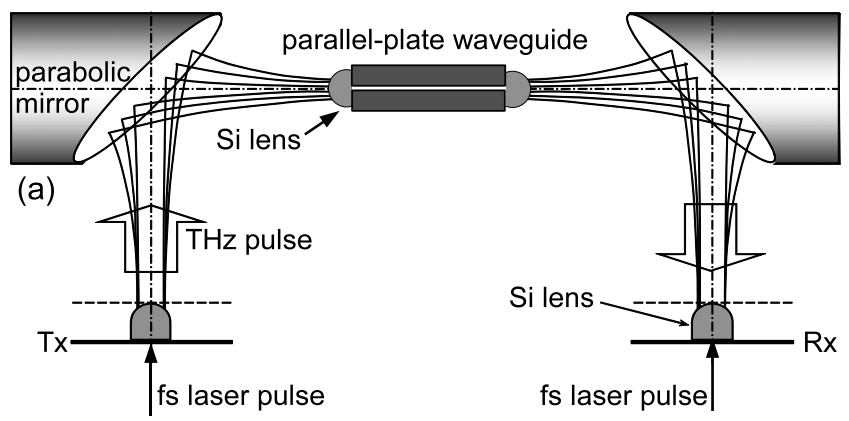

(b)

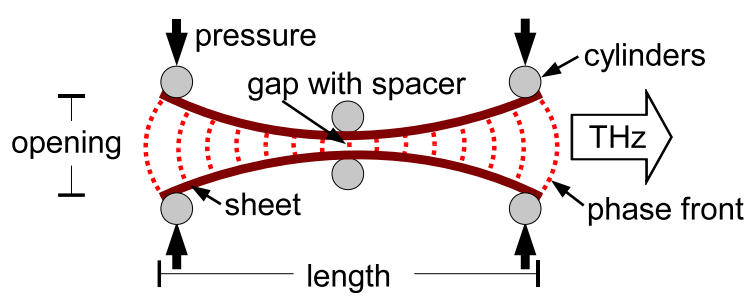

FIG. 1. (Color online) (a) THz-TDS experimental setup with a parallel-plate waveguide in the beamwaist. Si lenses couple the free-space radiation into the gap between the plates. (b) Metal flare assembly. When placed in the THz-TDS system, the smoothly curved metal flare guides the $\mathrm{THz}$ beam through the gap. Efficient input and output coupling are achieved. 
sponding to an intensity reduction by the factor of 4 . By the introduction of the confocal lenses and their imaging capabilities the coupling ratio is further reduced by 0.8 . The waveguide contributes losses by modal mismatch and Ohmic losses (0.8). The reported value of the overall amplitude coupling ratio using silicon optics and a $100 \mu \mathrm{m}$ waveguide is $32 \%{ }^{7}$ In our experiment, we replace the parallel-plate waveguide with the flare assembly and measure the coupling ratio through a gap with the same aperture dimension as typical waveguides.

For our measurements the flare assembly [Fig. 1(b)] replaces the parallel-plate waveguide at the beamwaist of the THz-TDS system. The metal sheets are mounted similar to an electric-field horn for the horizontally (i.e., in plane) polarized beam. They form an entrance and exit flare with a narrow gap at the center axis. The expected phase fronts of the free-standing flare are indicated as dashed lines copying the principle known from horn antennae. The actual phase fronts at the entrance and exit of the flare are an intermediate between the ones of the undisturbed $\mathrm{THz}$ system and the flare. Variable spacers outside of the $\mathrm{THz}$ beam can control the preset minimal distance. The metal sheets $(100 \mu \mathrm{m}$ thick copper and $35 \mathrm{~mm}$ wide) are naturally curved having an optical quality overall. This tendency to retain in a round shape without external force is used to form a very smooth flare with adjustable curvature. A transfer of the flare curvature into discrete coordinates was carried out for the initial and the pressed shim. Data analysis shows that to a good approximation a quadratic opening from the midpoint can be assumed for both of the flares. This is also expected because the flare under force should form a hyperbolic catenary, whose series expansion is quadratic in the first term.

The curvature of the flare is changed by symmetrically and gently pressing the four supporting rods at the end edges. The rods have no connection to the sheet, only a light contact. The center part is kept at a defined spacing by mechanical force to retain the preset gap, independent of curvature. This guarantees a smooth shape and allows for a steady tuning of the curvature and the flare's phase fronts. Furthermore, the surfaces change adiabatically with respect to the wavelength. ${ }^{7}$ This is very important to reduce reflection losses which occur at sharp edges or abrupt beam manipulations. The compression and expansion of the accepted $\mathrm{THz}$ beam, by the clear opening of the flare, is carried out over a distance of $4 \mathrm{~cm}$ or $6 \mathrm{~cm}$, corresponding to a total flare length of $8 \mathrm{~cm}$ or $12 \mathrm{~cm}$, respectively. This geometry extending over several hundred wavelengths maintains an adiabatic shape without beam perturbations. The full opening distance of the flare shown in Fig. 2 was controlled using linear stages. In terms of coupling ratio, the flare assembly is comparable to the standard parallel-plate waveguide with the same separation. But here, the sample can be directly located on the sheet.

For the measurements both flare full openings are symmetrically varied between 0 (contact) and $25 \mathrm{~mm}$. The measured coupling ratios evaluated in the time domain are shown in Fig. 2. For a Gaussian $\mathrm{THz}$ beam and at the optimized curvature the coupling ratio of the electric field through a $100 \mu \mathrm{m}$ gap is $18 \%$ for an $8 \mathrm{~cm}$ and $31 \%$ for a $12 \mathrm{~cm}$ long flare.

Starting from the full $25 \mathrm{~mm}$ opening of the $12 \mathrm{~cm}$ flare, as the opening is reduced the coupling ratio slightly increases up to a maximum around $12.5 \mathrm{~mm}$ until sharply decreasing

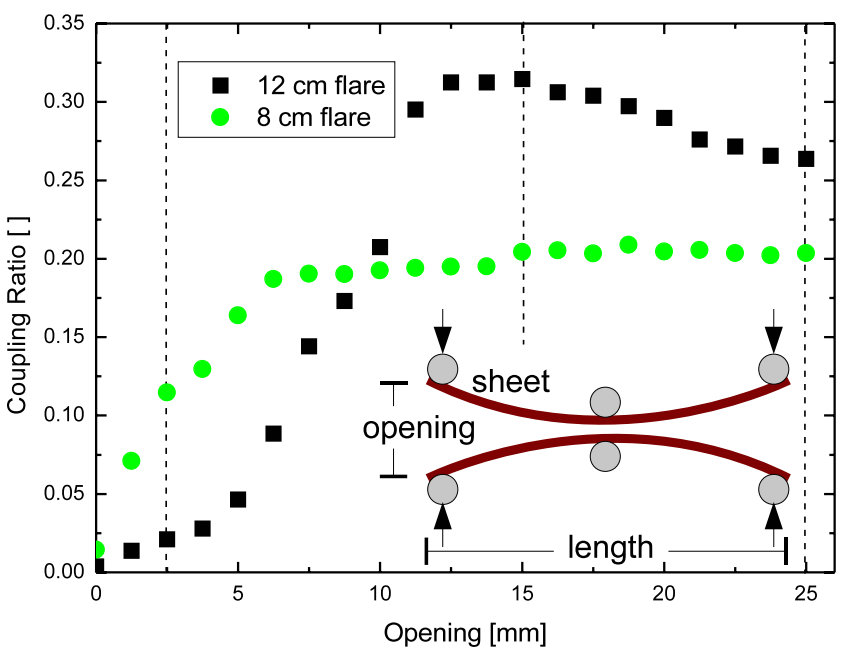

FIG. 2. (Color online) Measured amplitude transmission for a copper flare of variable full opening. An optimal opening occurs where the coupling ratio is the maximum. The longer and thereby smoother $12 \mathrm{~cm}$ flare has the higher coupling ratio. Inset: zoom on the geometry and the nomenclature of the flare.

at $10 \mathrm{~mm}$ opening because of beam truncation until vanishing at contact (zero opening). This behavior clearly indicates that there is an optimal intermediate shape for the flare. For large openings, the curvature change (especially at the outer part of the $\mathrm{THz}$ beam) is rather large (nonadiabatic), giving rise to reflection losses. Openings smaller than $10 \mathrm{~mm}$ cannot accept the larger beam into the horn. The coupling for small openings is further reduced by waveguidelike losses due to a longer propagation path through a compressed flare in the vicinity of the minimum gap. This effect has more influence for long flares than for short flares.

Within the measurement accuracy the coupling ratio for different openings of the $8 \mathrm{~cm}$ flare is roughly constant between 5 and $25 \mathrm{~mm}$. Assuming a $9 \mathrm{~mm}$ wavelength dependent $\mathrm{THz}$ spotsize at the entrance of the flare, the coupling starts to drop at smaller openings compared to the $12 \mathrm{~cm}$ flare. This may be an indication that the coupling is more efficient at the center of the flare. The further the beam edge is located away from the center axis of the flare, the less efficiently radiation can be guided into the horn at large openings due to the strong curvature and for small openings due to beam truncation.

The spectral evaluation for some selected flare openings (vertical lines in Fig. 2) are shown in Fig. 3. The solid lines mark the $12 \mathrm{~cm}$ and the dashed lines the $8 \mathrm{~cm}$ long flare, respectively. Certainly the overall coupling follows the behavior expected according to the amplitude transmission. It is remarkable that the coupling ratios for the appropriate shapes $(15$ or $25 \mathrm{~mm}$ ) are roughly constant as a function of frequency. This indicates that this type of coupling is not wavelength dependent, which is important for sensor technology. For small flare openings the higher frequencies couple less efficiently. The flare length changes the coupling ratio but does not influence the spectral characteristic.

The theoretical limit of coupling between the Gaussian beam of the $\mathrm{THz}$ emitter and the transverse electromagnetic (TEM)-mode of the corresponding waveguide for the flare is given by their overlap integral. Even with an optimal shape of the flare and phase front, the calculated amplitude transfer for a Gaussian THz beam (1/e amplitude width $4.5 \mathrm{~mm}$ ) into 


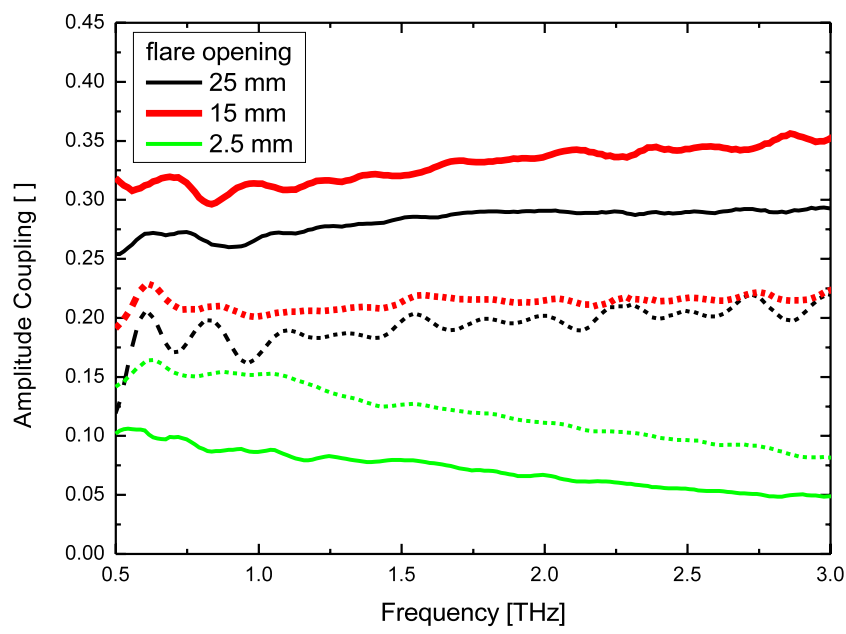

FIG. 3. (Color online) Amplitude transmission of broadband THz radiation measured for different flare openings (solid line: $12 \mathrm{~cm}$ flare and dashed: 8 $\mathrm{cm}$ flare). All flares show only a negligible spectral dependency of coupling which is desired for spectroscopic applications.

the lowest order mode of the supporting TEM waveguide is $89 \%$. This is calculated for a flare opening of $9 \mathrm{~mm}$. For the output, the same coupling is obtained, giving an optimal throughput amplitude coupling ratio of 0.79 . This, together with maintaining the adiabatic approximation, are the fundamental limiting factors. So, the results obtained with a $12 \mathrm{~cm}$ long flare still have potential for an improvement by a factor of more than 2 (Note added after submission: Our latest results showed a coupling ratio of 0.50 , achieved by changing the flare shape from quadratic to a double S). Considering the coupling reported using Si lenses, the coupling is excellent with respect to the overlap integral at the expense of Fresnel loss of 0.50 .

Compared to the results of Kim et al. ${ }^{8}$ obtained with bulk milled flares (coupling ratio of $56 \%$ at $1 \mathrm{THz}$ and dropping with higher frequencies) our results show a reasonable performance. We believe that our approach, relying only on naturally curved surfaces is advantageous because it is much easier to apply, does not need high precision milling and guarantees a steady smooth surface quality. Also regarding theoretical considerations, the circular geometries for the flare shape are preferable especially because of their much smaller geometrical extensions and reduced reflection. Finally, the expected maximum accessible field concentration for small spacings is higher for curved surfaces than for linear wedges. ${ }^{10}$

As the $\mathrm{THz}$ frequency range is located between the optical and the microwave regime, various fundamental $\mathrm{THz}$ experiments can be carried out using available optical components and keeping the experimental dimensions small compared to the microwaves. The horn-type coupling known from the millimeter waves offers a lot of advantages in the $\mathrm{THz}$ range. In particular, coupling by metallic optics is dispersionless because metals are nearly perfect reflectors below $3 \mathrm{THz}$. This fact can be used for coupling schemes to eliminate the reflections of transmissive optics in the $\mathrm{THz}$ beam. A long scan in the time domain shows that the flare does not introduce any observable reflections. This is very important for high-resolution waveguide spectroscopy where a long scanning range is needed.

We have shown that naturally curved metal flares with a length of several hundred wavelengths can be applied to efficiently couple broadband $\mathrm{THz}$ radiation through a subwavelength gap. The flares act like an adiabatic E-field horn on the collector as well as on the emitter side. Further experiments will prove the performance of this approach on particular samples with respect to minimum gap size, bandwidth, and resolution, in comparison with an extended parallel-plate waveguide. For real world application this technique, based on low-cost disposable components while keeping good results is promising.

This work was partially supported by the Fraunhofer Gesellschaft FhG internal program for the scientific exchange (Prof.x2) and the National Science Foundation.

${ }^{1}$ Y. Watanabe, K. Kawase, T. Ikari, H. Ito, Y. Ishikawa, and H. Minamide, Appl. Phys. Lett. 83, 800 (2003).

${ }^{2}$ J. Chen, Y. Chen, H. Zhao, G. J. Bastiaans, and X.-C. Zhang, Opt. Express 15, 12060 (2007)

${ }^{3}$ S. Wohnsiedler, M. Theuer, M. Herrmann, S. Islam, J. Jonuscheit, R. Beigang, and F. Hase, Proc. SPIE 7215, 72150H (2009).

${ }^{4}$ J. Zhang and D. Grischkowsky, Opt. Lett. 29, 1617 (2004).

${ }^{5}$ G. Gallot, S. Jamison, R. McGowan, and D. Grischkowsky, J. Opt. Soc. Am. B 17, 851 (2000).

${ }^{6}$ R. Mendis and D. Grischkowsky, Opt. Lett. 26, 846 (2001).

${ }^{7}$ J. Zhang and D. Grischkowsky, Appl. Phys. Lett. 86, 061109 (2005).

${ }^{8}$ S.-H. Kim, E. S. Lee, Y. B. Ji, and T.-I. Jeon, Opt. Express 18, 1289 (2010).

${ }^{9}$ M. van Exter and D. Grischkowsky, IEEE Trans. Microwave Theory Tech. 38, 1684 (1990).

${ }^{10}$ N. A. Janunts, K. S. Bagdasaryan, and K. V. Nerkararyan, Phys. Lett. A 269, 257 (2000). 